\title{
OPEN LEAVES IN CLOSED 3-MANIFOLDS
}

\author{
BY JOHN CANTWELL AND LAWRENCE CONLON ${ }^{1}$ \\ Communicated by Hugo Rossi, November 4, 1975
}

Let $N$ be an open, connected, orientable surface. Let $E(N)$ denote the set of ends of $N$, a compact, totally disconnected, separable space of ideal points at infinity [1]. The closed subset $E^{*}(N)$ of nonplanar ends consists of those ends that are limit points for sequences of handles of $N$. The topological pair $(E(N)$, $\left.E^{*}(N)\right)$ and the genus of $N$ determine the surface up to homeomorphism [4] , [8] .

Let $E^{(0)}(N)$ coincide with $E(N)$ and let $E^{(r)}(N)$ be the set of accumulation points of $E^{(r-1)}(N), r \geqslant 1$.

Given $N$, let $N^{(0)}$ coincide with $N$ and, if $r \geqslant 1$, obtain $N^{(r)}$ from $N^{(r-1)}$ by deleting a closed discrete set of points approaching all of the ends of $N^{(r-1)}$. Thus $E^{(r)}\left(N^{(r)}\right)=E(N)$.

Definition. The surface $N$ is of (finite) type $r \geqslant 0$ if $E^{(r)}(N) \neq \varnothing=$ $E^{(r+1)}(N)$.

The surfaces of finite type form a large and interesting class. There are basic type $r$ examples $N_{r, i}, 0 \leqslant i \leqslant q(r)<\infty$, such that all surfaces of finite type are finite connected sums of these basic ones and a compact surface. (The function $q(r)$ is amusing: $q(0)=1, q(1)=3, q(2)=(19), q(3)>10^{6}, q(4)>10^{300,000}$.) This makes possible several constructions and arguments that depend inductively on the type $r$.

Thus, for instance, a result of J. Sondow [9, p. 623] for surfaces of type 0 is improved and generalized as follows.

THEOREM 1. Every $N$ of finite type is homeomorphic to a leaf (a proper leaf if $N \neq\left(\mathbf{R}^{2}\right)^{(r)}, r \geqslant 1$ ) of a transversely orientable $C^{\infty}$ foliation in a suitable closed orientable 3-manifold. Furthermore, every $N$ of finite type is homeomorphic to a proper leaf of a transversely orientable $C^{\mathbf{1}}$ foliation in every closed 3manifold.

We remark that the $C^{1}$ construction in the proof of this theorem provides a negative answer to a question of T. Nishimori [6, Problem 6.4].

The following greatly generalizes results of S. Goodman [2], [3, §3] .

THEOREM 2. Let $N$ be of finite type and let $M$ be a closed 3-manifold. Then

AMS (MOS) subject classifications (1970). Primary 57D30; Secondary 57A05, 57A10.

${ }^{1}$ Supported in part by NSF Grant no. MPS 74-07068 A01. 
(a) if $N$ has no isolated nonplanar end, it is homeomorphic to a leaf (proper if $N \neq\left(\mathbf{R}^{2}\right)^{(r)}, r \geqslant 1$ ) of a transversely orientable $C^{\infty}$ foliation of $M$;

(b) if $N$ has only nonplanar ends or is the connected sum of such a surface with one as in (a), and if $M$ is orientable and is not a rational homology sphere, then $N$ is homeomorphic to a proper leaf of a transversely orientable $C^{\infty}$ foliation of $M$.

Remark that every surface as in (b) has at least one isolated nonplanar end.

By [10] and [11] smooth transversely orientable foliations exist on every closed 3-manifold. Our theorems are proven by constructions for modifying such foliations along a closed transversal, so as to produce the prescribed leaf $N$. These constructions prove more than was asserted. Indeed, the foliations can be kept free of exceptional minimal sets and the leaf homeomorphic to $N$ always has polynomial growth [7]. Most of our restrictive hypotheses are necessary if such nice asymptotic behavior is to be preserved. Indeed,

THEOREM 3. If $r \geqslant 1$, then $\left(\mathbf{R}^{2}\right)^{(r)}$ cannot occur as a proper leaf with nonexponential growth in a $C^{2}$ foliation of any closed 3-manifold. These are the only surfaces of finite type for which this assertion is true.

THEOREM 4. Let L be a proper leaf of a transversely orientable $C^{2}$ foliation of a rational homology 3-sphere. If $L$ has an isolated nonplanar end, then $L$ has exponential growth and approaches an exceptional minimal set.

These two results rely heavily on work of J. Plante [7] and a lemma of N. Kopell [5, Lemma 1]. Such nontrivial interplay between the topology of a leaf and its asymptotic behavior seems surprising.

We also know a class of surfaces of infinite type, all having a Cantor subset of ends, each of which occurs as a leaf of a $C^{\infty}$ foliation in every closed 3 manifold.

The proofs of these results will appear elsewhere.

\section{REFERENCES}

1. L. V. Ahlfors and L. Sario, Riemann surfaces. Chap. I. Section 6, Princeton Math. Ser., no. 26, Princeton Univ. Press, Princeton, N. J., 1960. MR 22 \#5729.

2. S. E. Goodman, Closed leaves in foliated 3-manifolds, Proc. Nat. Acad. Sci. U.S.A. 71 (1974), 4414-4415. MR 50 \#3243. appear).

3. - Closed leaves in foliations of codimension one, Comment Math. Helv. (to

4. B. Kerékjártó, Vorlesungen über Topologie. I, Springer, Berlin, 1923.

5. N. Kopell, Commuting diffeomorphisms, Proc. Sympos. Pure Math., vol. 14, Amer. Math. Soc., Providence, R. I., 1970, pp. 165-184. MR 42 \#5285.

6. T. Nishimori, Isolated ends of open leaves of codimension-one foliations, Quart. J. Math. Oxford (3) 26 (1975), 159-167.

7. J. Plante, Foliations with measure preserving holonomy, Ann. of Math. (to appear).

8. I. Richards, On the classification of noncompact surfaces, Trans. Amer. Math. Soc. 106 (1963), 259-269. MR 26 \#746.

9. J. Sondow, When is a manifold a leaf of some foliation? Bull. Amer. Math. Soc. 81 (1975), 622-624. 
10. W. Thurston, A local construction of foliations for three-manifolds, Proc. Sympos. Pure Math., vol. 27, Amer. Math. Soc., Providence, R. I., 1975, pp. 315-319.

11. J. W. Wood, Foliations on 3-manifolds, Ann. of Math. (2) 89 (1969), 336-358. MR $40 \# 2123$.

DEPARTMENT OF MATHEMATICS, ST. LOUIS UNIVERSITY, ST. LOUIS, MISSOURI 63103

DEPARTMENT OF MATHEMATICS, WASHINGTON UNIVERSITY, ST. LOUIS, MISSOURI 63130 